\title{
Estimation of the power of geoacoustic emission sources registered in Mikizha Lake, Kamchatka region
}

\author{
Albert Shcherbina ${ }^{1,1}$, and Alexandra Solodchuk ${ }^{1}$ \\ ${ }^{1}$ Institute of Cosmophysical Research and Radio Wave Propagation FEB RAS, Laboratory \\ of Acoustic Research, Mirnaya str., 7, Paratunka, Kamchatskiy kray, 684034, Russia
}

\begin{abstract}
A method for estimating the power of geoacoustic emission sources recorded by a combined hydroacoustic receiver in Mikizha lake (Kamchatka region) is proposed. The results of the method application at different stages of seismic activity are presented.
\end{abstract}

\section{Introduction}

It is known that strong earthquakes can be accompanied by rocks deformation, noticeable at a distance of hundreds of kilometers from their epicenter [1]. As a result of long-term observations of geoacoustic emission in Kamchatka, it was found that an increase in the deformation rate of rock massifs leads to an increase in the intensity of high-frequency geoacoustic emission [2]. This effect is determined by rock deformations at observation points and is most pronounced in the kilohertz frequency range 1-3 days before earthquakes [3]. Power estimation of geoacoustic emission sources will be useful for the estimation of energy characteristics of rocks stress-strain state in an observation point.

\section{Power estimation}

The problem of determining a spherical wave source power is solved quite simply. An amount of sound energy $W$ equal to the source acoustic power will flow through a spherical surface with area $S$ and radius $r$ per time unit [4]. In the case of a spherical wave, a sound wave with an average intensity $J_{\mathrm{s}}$ will pass through a unit of surface area:

$$
J_{\mathrm{s}}=\frac{W}{S} .
$$

Herein, the intensity of the sound and its power are determined by averaging over one oscillation period.

\footnotetext{
${ }^{1}$ Corresponding author: albert_pkam@mail.ru
} 
The sound wave intensity $J_{\mathrm{r}}$ at the receiving point is defined as

$$
J_{\mathrm{r}}=\frac{1}{2} P_{0} V
$$

where: $P_{0}, V$ are the pressure and the oscillatory speed, respectively.

The sound wave intensity at the receiving point can be expressed in terms of the sound power:

$$
W_{\mathrm{r}}=J_{\mathrm{r}} S_{r},
$$

where: $S_{r}$ is the receiver cross-sectional area in a plane perpendicular to the wave arrival direction.

In the case when the radius $r$ of the sound wave front is equal to the distance from the source to the receiver, the following expressions will be valid:

$$
\begin{gathered}
J_{\mathrm{r}}=J_{\mathrm{s}}, \\
\frac{W_{\mathrm{r}}}{S_{\mathrm{r}}}=\frac{W}{S}, \\
W=W_{\mathrm{r}} \frac{S}{S_{\mathrm{r}}} .
\end{gathered}
$$

Thus, by registering a part of the sound radiation power $W_{\mathrm{r}}$ at the receiving point and knowing the distance from the source to the receiver, it is possible to determine the source power by first determining the surface area $S$ of the sound wave front.

In the area of geoacoustic emission radiation, shear sources of acoustic emission predominate, because the rock strength with respect to tangential stresses is less than compressive. It was shown in $[5,6]$ that the orientation of shear sources is determined by the direction of the maximum tangential stresses, oriented mainly at an angle of $45^{\circ}$ to the axis of greatest compression. The maxima of the longitudinal acoustic oscillations occur on the directions perpendicular to this axis (Fig. 1a) [7]. Transverse oscillations can be excluded from consideration, since the receiver is located in water environment.

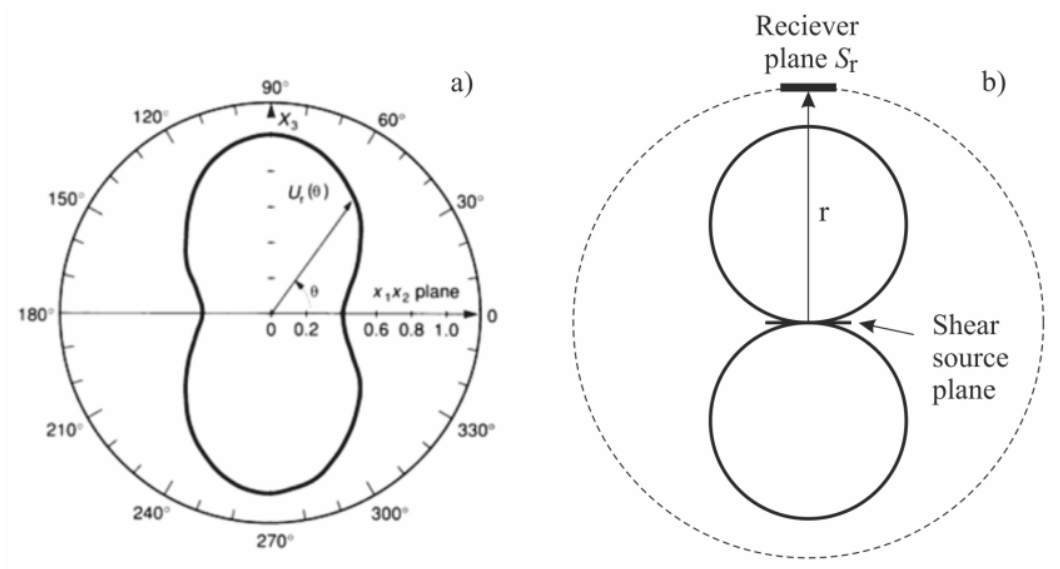

Fig. 1. Radiation pattern of acoustic emission of a shear source (a) and its approximation (b). The dashed line indicates the front of the sound wave. 
The sound wave intensity will vary depending on the angle between the signal arrival direction and the shear plane in the case of a shear signal source with an uneven radiation pattern. The calculation of the power of such a source is complicated. The power of a source can be estimated as

$$
W=\iint J(\alpha, \varphi) d \alpha d \varphi
$$

To obtain an upper estimate of the source radiation power, authors assume that the acoustic emission radiation pattern of the source looks like Fig. 1b. Also authors assume that the receiver registers the power from the direction with the maximum radiation level. Then the radiation power of the source can be calculated as

$$
W=2 \pi \int \arccos ^{2}\left(\frac{x}{J_{\mathrm{r}}}\right) d x .
$$

Calculating the integral, we obtain the ratio:

$$
W=2 \pi J_{\mathrm{r}}(\pi-2)
$$

The calculation of the sound intensity at the receiving point was carried out according to Eq. 2 by averaging the signals for a time $T$ equal to or exceeding the wave period from one to three times in the pulse section with the largest amplitude (Fig. 2). The remaining part of the pulse, as a rule, does not have a clear periodicity and is significantly smaller in amplitude than the main part of the signal. The contribution of the pulse attenuation section to the total intensity is not significant, therefore, it was not included in the calculation.

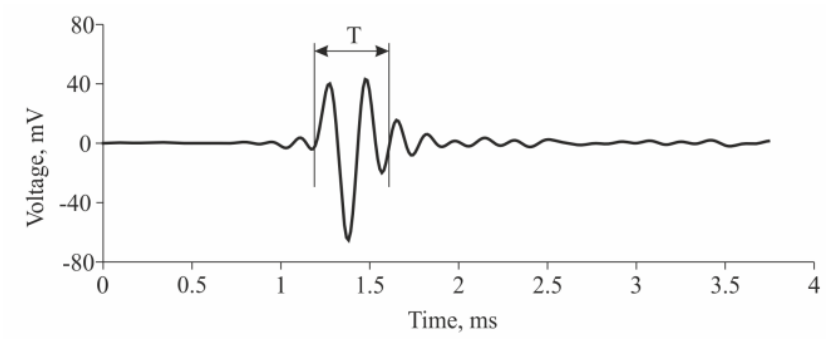

Fig. 2. Waveform of geoacoustic emission. $T$ is signal averaging time.

As mentioned above, to calculate the source power, information about the distance to it is necessary. The distance can be obtained by analyzing the phase portrait of the recorded signal. Under certain conditions of propagation and registration of the signal, it is possible to distinguish a characteristic change in the medium particle motion phase due to the influence of the wave part reflected from the surface. In the conditions of location of the combined receiver by the bottom of a shallow lake, there is often a summation of the signal transmitted directly from the source and its reflection from the water surface or the ice lower edge.

It is clear from the example (Fig. 3) that during the initial phase of the recorded pulse, reciprocal motion of the receiver along the axis, directed to the signal source, is observed. About $0.5 \mathrm{~ms}$ after it, significant change of the hodograph is observed. It is caused by the overlapping of the signal reflected vertical component on the main signal. These features of the recorded signal may be used to determine the distance to its source. 

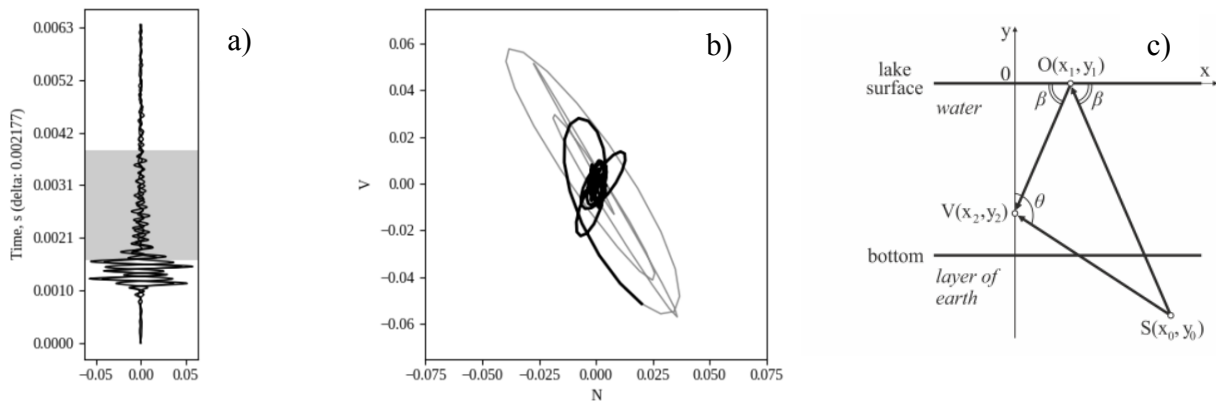

Fig. 3. Example of geoacoustic pulse recorded together with the reflected signal (a) and its reflection in a vertical plane parallel to the North-South axis (b). The gray area (a) and a solid line (b) is a signal fragment formed by the direct and reflected waves. In figure $3 \mathrm{c}$ it is scheme of direct wave (SV) and reflected wave (OV) propagation; $S\left(x_{0}, y_{0}\right)$ is the source, $O\left(x_{1}, y_{1}\right)$ is the incidence point, $V\left(\mathrm{x}_{2}, \mathrm{y}_{2}\right)$ is the receiver.

The distance to the signal source will correspond to the vector SV length, depending on the geometric dimensions of the system and the delay between the moments of registration of the direct and the reflected waves [8]. The sound wave refraction effects at the medium boundary are not taken into consideration. We should note, that in our conditions the consideration of the refraction effects will not give an appreciable increase in accuracy, since the signal sources distances are comparable with their linear dimensions [9].

It should be noted, that during the propagation the sound wave passes part of the path through sedimentary rocks (Fig. 3c). The absorption coefficient in sedimentary rocks is estimated from $0.17 \mathrm{~dB} / \mathrm{m}$ at $1 \mathrm{kHz}$ to $4 \mathrm{~dB} / \mathrm{m}$ at $10 \mathrm{kHz}[10]$.

\section{Results of the power estimation}

Table 1. Power of geoacoustic emission sources during different stages of seismic activity.

\begin{tabular}{|l|c|c|c|c|c|}
\hline № & Date, time & $\begin{array}{c}\text { Azimuth, } \\
\text { degrees }\end{array}$ & $\begin{array}{c}\text { Elevation angle, } \\
\text { degrees }\end{array}$ & $\begin{array}{c}\text { Distance } \\
\text { to source, } \mathrm{m}\end{array}$ & Power, W \\
\hline 1 & $05.12 .20140: 12$ & 11 & 155 & 0.87 & $1.36 \cdot 10^{-10}$ \\
\hline 2 & $05.12 .20140: 48$ & 11 & 155 & 5.10 & $1.72 \cdot 10^{-9}$ \\
\hline 3 & $05.12 .20141: 03$ & 162 & 140 & 0.80 & $4.59 \cdot 10^{-13}$ \\
\hline 4 & $05.12 .20147: 32$ & 20 & 156 & 3.71 & $9.34 \cdot 10^{-10}$ \\
\hline 5 & $05.12 .20147: 40$ & 20 & 156 & 4.00 & $1.19 \cdot 10^{-9}$ \\
\hline 6 & $05.12 .20148: 42$ & 15 & 150 & 1.70 & $4.79 \cdot 10^{-10}$ \\
\hline 7 & $05.12 .201415: 29$ & 20 & 153 & 4.70 & $5.59 \cdot 10^{-10}$ \\
\hline 8 & $08.12 .201414: 09$ & 356 & 150 & 2.30 & $4.63 \cdot 10^{-5}$ \\
\hline 9 & $08.12 .201414: 31$ & 270 & 138 & 1.60 & $3.17 \cdot 10^{-5}$ \\
\hline 10 & $08.12 .201415: 18$ & 274 & 137 & 2.30 & $9.91 \cdot 10^{-6}$ \\
\hline 11 & $08.12 .201415: 19$ & 274 & 137 & 8.20 & $7.54 \cdot 10^{-5}$ \\
\hline
\end{tabular}

Using the proposed method, the power of geoacoustic signal sources was estimated in a background period (Table 1, lines 1 through 7) and during earthquake development 
process (Table 1, rows 8 through 11). The earthquake occurred 08.12.2014 at 9:52 UT with magnitude $M=5.6$ (energy class 12.8) at the epicentral distance of $303 \mathrm{~km}$ with the azimuth $179^{\circ}$, the coordinates of the hypocenter was $50.26^{\circ} \mathrm{N}, 158.27^{\circ} \mathrm{E}$, depth $66.25 \mathrm{~km}$. Earthquake characteristics were taken from the operational catalogue of the Kamchatka branch of the Federal Research Center Geophysical Survey of the Russian Academy of Sciences. A combined receiver, produced by ZAO «Geoakustika» at FGUP VNIIFTRI, is applied to record geoacoustic signals. The operating frequency range of the device is from 5 to $11000 \mathrm{~Hz}$, the sensitivity with preliminary amplifications of the omnidirectional pressure channel of $500 \mathrm{mV} \cdot \mathrm{Pa}^{-1}$, the sensitivity of the vector channels increases by $6 \mathrm{~dB}$ per octave and amounts to $20 \mathrm{mV} \cdot \mathrm{Pa}^{-1}$ at the frequency of $10 \mathrm{kHz}$.

The average value power of the geoacoustic signal sources power in the background period was $7.43 \cdot 10^{-10} \mathrm{~W}$, which is several orders of magnitude less than that during seismic activity $\left(4.08 \cdot 10^{-5} \mathrm{~W}\right)$.

The work was carried out by the means of the Common Use Center "North-Eastern Heliogeophysical Center "CKP_558279".

\section{References}

1. A.V. Kalinina, V.A. Volkov, R. Vieira, J. Arnoso, A.M. Buloshnikov, A.V. Gorbatikov, A.V. Nikolaev, J. Volcanol. Seismol., 1, 46 (2010)

2. Yu.V. Marapulets, Bulletin KRASEC. Phys. \& Math. Sci., 10(1), 39 (2015)

3. G.I. Dolgikh, A.V. Kuptsov, I.A. Larionov, Yu.V. Marapulets, V.A. Shvets, B.M. Shevtsov, O.P. Shirokov, V.A. Chupin, S.V. Yakovenko, Dokl. Earth Sc., 413, 281 (2007)

4. A.M. Turin, A.P. Stashkevich, E.S. Taranov, Basics of hydroacoustics (Sudostroenie, Leningrad, 1966) 294

5. S.D. Vinogradov, Izvestiya of the Academy of Sciences of the USSR. Physics of the Solid Earth, 7, 20 (1976)

6. O.G. Shamina, V.I. Ponyatovskaya, Model studies of heterogeneous and fractured media, 179 (IFZ RAN, Moscow, 1993)

7. A.A. Pollock, Acoustic emission inspection (Technical Report TR-103-96-12/98, Physical Acoustics Corporation, 1989)

8. A. Shcherbina, A. Solodchuk, E3S Web of Conf., 62, 03004 (2018)

9. Yu.V. Marapulets, O.O. Lukovenkova, A.B. Tristanov, A.A. Kim, Methods for recording and for time-frequency analysis of geoacoustic emission signals, 148 (Dalnauka, Vladivostk, 2017)

10. I.S. Berzon, A.M. Epinateva, G.N. Pariyskaya, S.P. Starodubrovskaya, Dynamic characteristics of seismic waves in real environments, 512 (Publishing house of the USSR Academy of Sciences, Moscow, 1962) 